Check for updates

Cite this: Nanoscale Adv., 2019, 1, 195

\title{
Ultra-stable pulse generation in ytterbium-doped fiber laser based on black phosphorus $\dagger$
}

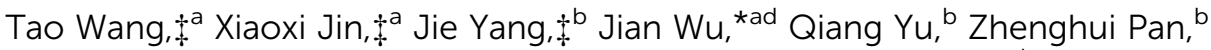 \\ Hanshuo Wu, ${ }^{a}$ Junzi Li, ${ }^{c}$ Rongtao Su, ${ }^{a}$ Jiangming $\mathrm{Xu}^{a}{ }^{a}$ Kai Zhang, ${ }^{\mathrm{b}}$ Tingchao He (D) *c \\ and Pu Zhou*a
}

We demonstrated a high-quality black phosphorus (BP) crystal fabricated via a modified electrochemical delamination exfoliation process. Employing the nonlinear transmittance method and Z-scan technique, the nonlinear optical properties of BP were characterized. Based on the saturable absorber (SA) of BP, we designed a passively Q-switched ytterbium (Yb)-doped fiber laser operating at $1.06 \mu \mathrm{m}$. Additionally, the pulse laser could operate stably for at least 69 days. These experimental results indicate that the modified BP is an ultra-stable and promising optical modulation material for ultrashort pulse generation in $\mathrm{Yb}$-doped fiber lasers.

Received 18th September 2018 Accepted 2nd December 2018

DOI: $10.1039 / c 8 n a 00221 e$

rsc.li/nanoscale-advances

visible to near-infrared region. Therefore, BP is an excellent SA for wide-band ultrafast photonics application.

Since the first BP-based SA (BP-SA) deposited around Recently, as saturable absorbers (SAs), two-dimensional (2D) materials such as graphene, ${ }^{1}$ carbon nanotubes, ${ }^{2}$ transitionmetal dichalcogenides (TMDs), ${ }^{3,4}$ and topological insulators $(\mathrm{TIs})^{5-7}$ have attracted much attention to generate pulse lasers. The passive ways based on SA to produce pulse lasers have the advantages of low cost, easy implementation and compactness. ${ }^{8}$ However, most 2D materials have some shortcomings that seriously limit their practical application. For example, graphene has a zero band gap, ${ }^{9}$ which limits its applications where strong interactions between the laser and material are needed, although it possesses wide-band absorption and ultrafast recovery time. TMDs have a wide band gap, but may be only suitable for absorption in the visible wavelength. Black phosphorus (BP) is another potential SA for ultra-short pulse generation, which is regarded as the most thermo-dynamically stable allotrope. ${ }^{10} \mathrm{BP}$ has a direct bandgap, which depends on the number of layers and ranges from $0.3 \mathrm{eV}$ in the bulk state to $2.0 \mathrm{eV}$ in the monolayer state. ${ }^{11-13}$ This can greatly enhance the interactions between light and $\mathrm{BP}^{14}$ at wavelengths from the

${ }^{a}$ College of Advanced Interdisciplinary Studies, National University of Defense Technology, Changsha, 410073, China. E-mail: wujian15203@163.com; zhoupu203@163.com

${ }^{b} i$-Lab, Suzhou Institute of Nano-Tech and Nano-Bionics, Chinese Academy of Sciences, Suzhou 215123, China

${ }^{c}$ College of Physics and Energy, Shenzhen University, Shenzhen 518060, China. E-mail: tche@szu.edu.cn

${ }^{d}$ CAS Key Laboratory of Nano-Bio Interface, Suzhou Institute of Nano-Tech and NanoBionics, Chinese Academy of Sciences, Suzhou 215123, China

$\dagger$ Electronic supplementary information (ESI) available. See DOI: 10.1039/c8na00221e

$\$$ Authors contributed equally. a microfiber was proposed, ${ }^{15}$ there has been much progress in the field of BP-based pulse laser generation. ${ }^{16-21}$ BP-SA can be manufactured via liquid phase exfoliation technology ${ }^{14}$ or the mechanical exfoliation method. ${ }^{22}$ Zhang et al. reported few-layer selenium-doped black phosphorus with high quality and low cost in $2017 .{ }^{23}$ However, avoiding oxidation and preventing optical damage to the BP-SA still limit its application. ${ }^{24}$ Research on the stability of BP-SA is scarce. M. Hisyam et al. observed a mode-locked pulse based on BP at 20 minute intervals for 80 minutes. ${ }^{16}$ Under $816 \mathrm{~mW}$ pump power, the peak amplitudes of the spectrum varied within $\pm 1 \mathrm{~dB}$ with a signal to noise ratio (SNR) of $47 \mathrm{~dB} .{ }^{16}$ Thus, further enhancing the long-term stability of $\mathrm{BP}$ is another obstacle that needs to be addressed.

Herein, BP was fabricated via a modified electrochemical delamination exfoliation process. ${ }^{25}$ Based on the BP-SA device, we demonstrated a passively Q-switched Yb-doped fiber laser operating in the $1.06 \mu \mathrm{m}$ region. A stable Q-switched pulse with a maximum output power of $12.6 \mathrm{~mW}$ and a slope efficiency of $11.1 \%$ WAS achieved. The laser could operate stably for at least 69 days, and its output properties were recorded in detail. To the best of our knowledge, our laser exhibits the best stability compared with that reported to date.

\section{Sample preparation and characterization of black phosphorus saturable absorber}

High quality and large-size BP bulk crystals were produced via a modified gas-phase transformation method ${ }^{23,26}$ (details in ESI section $1 \dagger$ ). 
For the exfoliation process, the non-oxidative electrochemical delamination strategy exfoliation process was performed through electrochemical engineering in non-aqueous electrolytes ${ }^{25}$ using a two-electrode system. About several hundred mg of bulk BP crystals was used as the cathode, and a piece of platinum foil was used as the anode. The electrolyte was made by dissolving tetra- $n$ butyl-ammonium bisulfate $\left(\mathrm{TBA} \cdot \mathrm{HSO}_{4}\right)$ in anhydrous deoxygenated propylene carbonate with the concentration of $0.1 \mathrm{M}$. The electrolysis voltage was set at $-30.0 \mathrm{~V}$ at the beginning of the delamination and was turned down to $-10 \mathrm{~V}$ after $20 \mathrm{~min}$.

For further characterization, some treatments were needed. The exfoliated BP was centrifuged at $8000 \mathrm{rpm}$ for $5 \mathrm{~min}$ and washed with anhydrous propylene carbonate and isopropyl alcohol (IPA) twice. Afterwards, a moderate amount of aswashed BP flakes were dispersed into anhydrous IPA by mild sonication for 15 min. Finally, the supernatant was decanted for further fabrication and characterization (Fig. 1(a)).

The atomic force microscopy (AFM) image of a typical sample and the associated height variations along the marked lines are depicted in Fig. 1(b). The thickness was measured to be 7-14 nm. The dominant BP film was observed using a Hitachi ${ }^{\circledR}$ S-4800 scanning electron microscope (SEM). The corresponding SEM image of a typical few-layer BP flake from the crystal on a silicon substrate is shown in Fig. 1(c). The microstructure and chemical composition of the BP crystal were thoroughly probed using a Tecnai ${ }^{\circledR}$ G2 F20 S-Twin transmission electron microscope (TEM), as shown in Fig. 1(d-f). A high-resolution TEM image of the micro-flake is shown in Fig. 1(e), which illustrated a clear lattice fringe with an interplanar space of $0.33 \mathrm{~nm}$. As shown in Fig. 1(f), the selected area electron diffraction pattern can be well
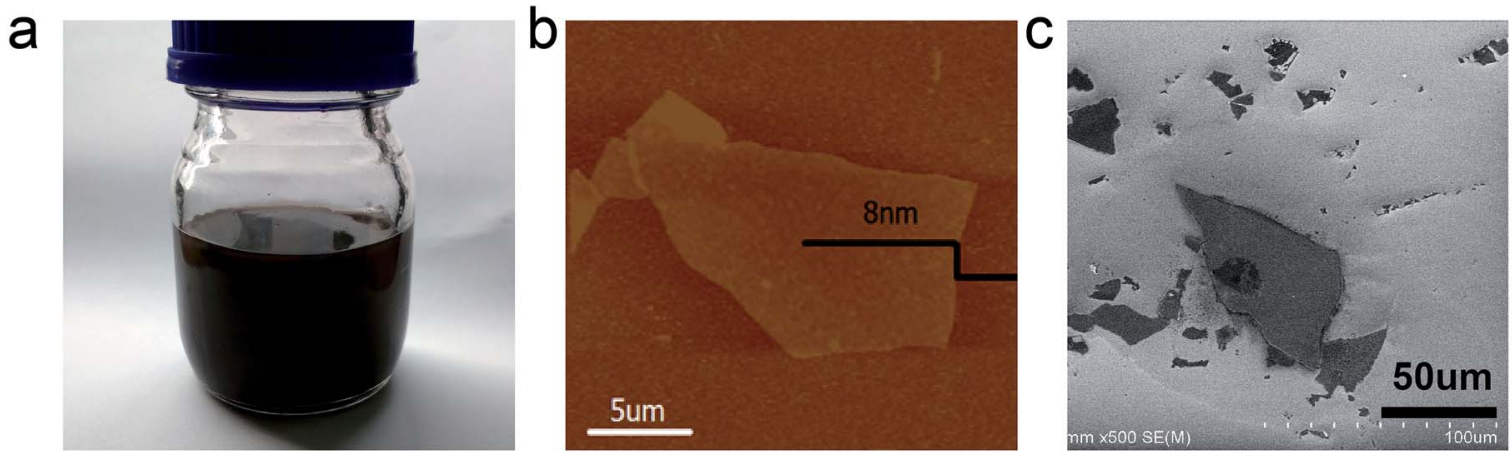

d

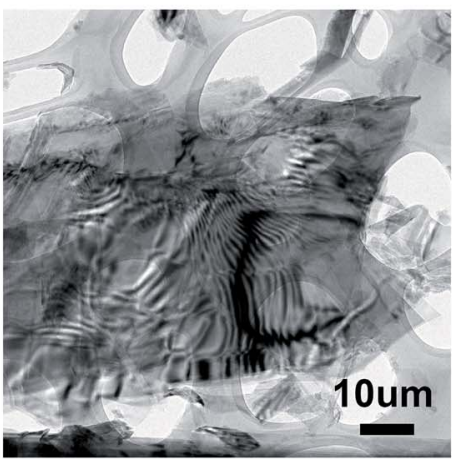

9

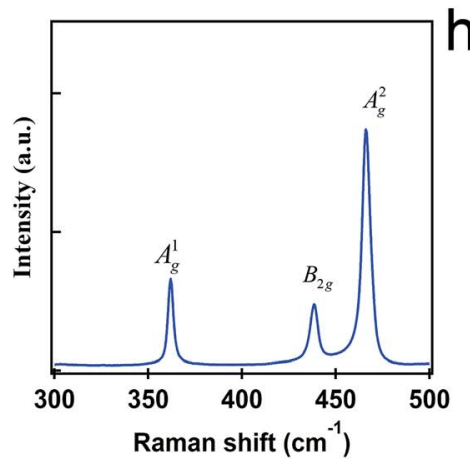

e
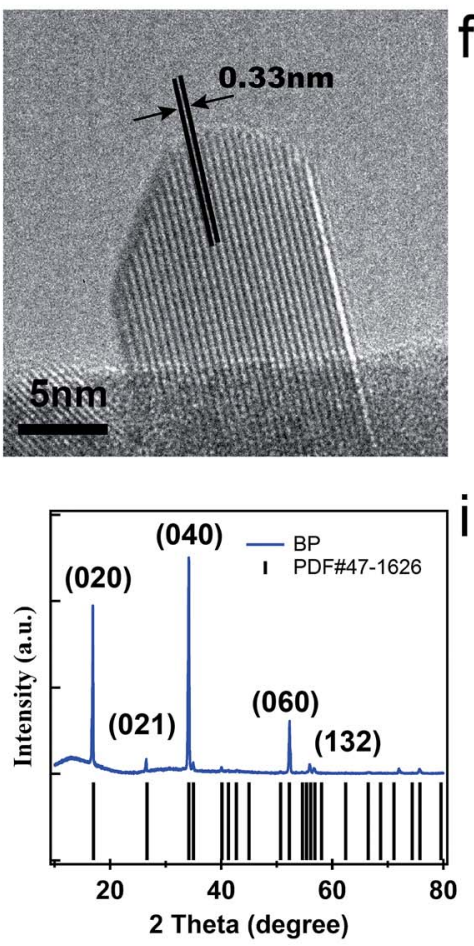
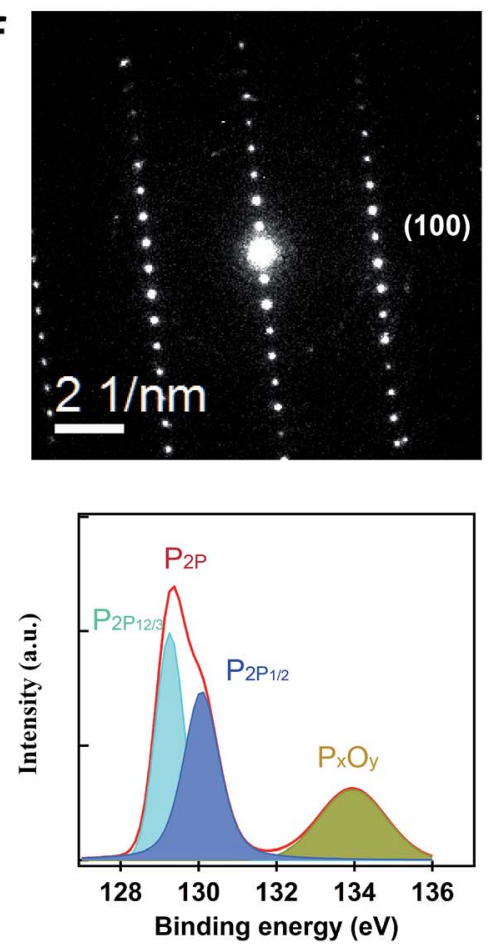

Fig. 1 Characteristics of the few-layer BP crystals synthesized via the electrochemical delamination exfoliation method: (a) digital image of the few-layer BP flakes ink, (b) atomic force microscopy (AFM) image of a typical few-layer BP flake from the crystal on a silicon substrate, (c) scanning electron microscopy (SEM) image of a typical few-layer BP flake from the crystal on a silicon substrate, (d) typical transmission electron microscopy (TEM) image of a BP flake, (e) high-resolution TEM image of a BP flake, (f) TEM neutron diffraction image of a BP flake, (g) Raman spectrum of the BP, (h) X-ray diffraction (XRD) pattern of the BP sample, which confirms its high crystallinity and (i) X-ray photoelectron spectroscopy (XPS) spectrum of the BP sample. 
indexed to an orthorhombic structure and the zone axis was determined as the [010] direction. In Fig. 1(g), the identity of the BP-SA was also verified by Raman spectroscopy, which discloses three characteristic vibration modes located at $362 \mathrm{~cm}^{-1}\left(\mathrm{~A}_{\mathrm{g} 1}\right)$, $437.5 \mathrm{~cm}^{-1}\left(\mathrm{~B}_{2 \mathrm{~g}}\right)$, and $466 \mathrm{~cm}^{-1}\left(\mathrm{~A}_{\mathrm{g} 2}\right)$. The integrated intensity ratio $A_{\mathrm{g}}{ }^{1} / A_{\mathrm{g}}{ }^{2}$ is 0.41 , which is greater than 0.20 and in the range of $0.40-0.60$, indicating its low oxidation level. ${ }^{27}$ The crystal structure of the BP-SA sample was characterized via X-ray diffraction (XRD). The XRD image exhibits high diffraction peaks in Fig. 1(h). The strong intensity diffraction peaks at $17.08^{\circ}, 34.28^{\circ}$, and $52.48^{\circ}$ correspond to the (020), (040), and (060) planes, respectively, which indicate that the BP sample possesses an orthorhombic structure with high crystalline quality. The X-ray photoelectron spectroscopy (XPS) spectrum of the BP crystal is shown in Fig. 1(i). The high-resolution XPS reveals that the band associated with $\mathrm{P}_{2 \mathrm{p}}$ appears at about $130 \mathrm{eV}$, which can be divided into $\mathrm{P}_{2 \mathrm{p} 1 / 2}(130.5$ $\mathrm{eV}$ ) and $\mathrm{P}_{2 \mathrm{p} 3 / 2}(129.5 \mathrm{eV})$, as the key feature of crystalline $\mathrm{BP}$. Moreover, the oxidized phosphorus peaks near $132 \mathrm{eV}$ disappear in the XPS spectra, indicating that the BP is well-prepared and free from oxidation during the exfoliation process.

\section{Results and discussions}

\subsection{Nonlinear optical properties of black phosphorus}

To prepare the SA film, the vacuum filtration method was used. The exfoliated BP solution was deposited onto a piece of filter
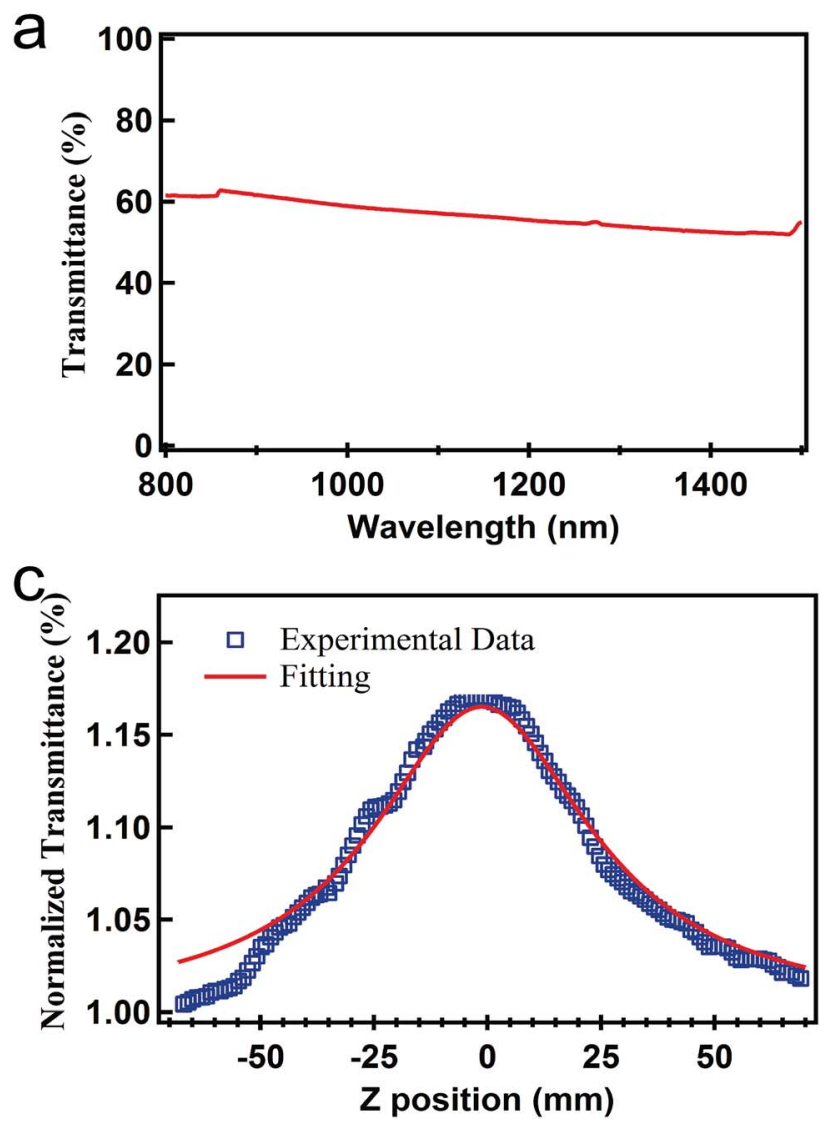

membrane via vacuum filtration. Finally, the sample was dried in a vacuum oven at $50{ }^{\circ} \mathrm{C}$ for $20 \mathrm{~min}$, and the thin film was peeled off with tweezers. The fiber ferrule with the deposited BPSA was then connected with a clean one via a fiber ferrule adapter, forming an SA ready for use in the fiber laser (see details in Fig. S1-S2 in the ESI $\dagger$ ).

Fig. 2(a) shows the linear transmittance spectrum of the BP film in the 800-1500 $\mathrm{nm}$ spectral range, which was measured using a home-built light source system. The transmittance was about $60 \%$ at $1064 \mathrm{~nm}$.

The nonlinear optical transmittance of BP was characterized under different input power intensities at the wavelength of $1060 \mathrm{~nm}(1000 \mathrm{~Hz}, 100 \mathrm{fs})$, as depicted in Fig. 2 (b). The relevant curve can be fitted by the following formula:

$$
T(I)=1-\frac{a_{\mathrm{s}}}{1+I / I_{\mathrm{sat}}}-a_{\mathrm{ns}}
$$

where, $T(I)$ is the transmittance, $a_{\mathrm{s}}$ is the modulation depth, $I$ is the incident power intensity, $I_{\mathrm{sat}}$ is the saturated intensity, and $a_{\mathrm{ns}}$ is the unsaturated loss. The corresponding modulation depth, unsaturated loss, and saturated intensity were fitted to be $15.6 \%$, $26.1 \%$, and $284 \mathrm{GW} \mathrm{cm}^{-2}$, respectively. We also characterized the nonlinear optical properties using the Z-scan technique. ${ }^{28}$

The open-aperture Z-scan results are shown in Fig. 2(c), and the normalized transmittance can be fitted by the following formula:

b
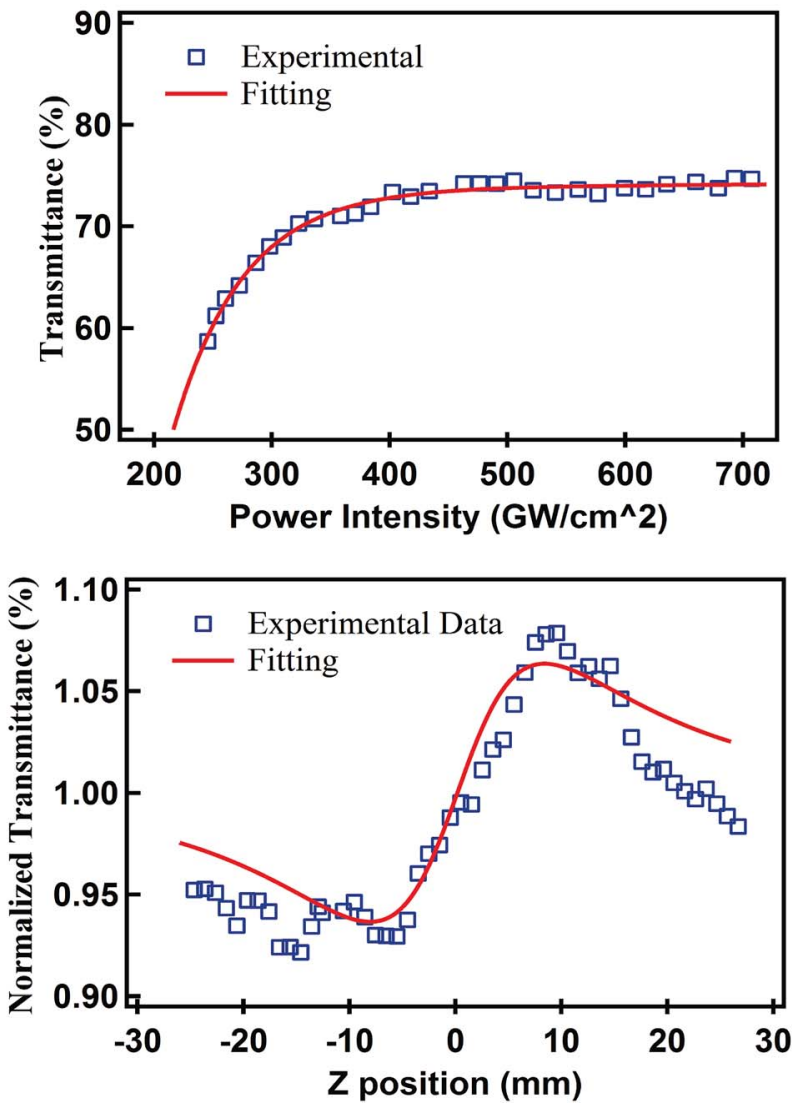

Fig. 2 (a) Optical transmittance for the BP, (b) relation between the incident intensity and transmittance of the BP crystal, (c) open Z-scan curve for the BP crystal, and (d) close/open Z-scan curve for the BP crystal. 


$$
T_{\text {norm }}(z)=1-\frac{\beta I_{0} L_{\text {eff }}}{2 \sqrt{2}\left(1+z^{2} / z_{0}^{2}\right)}
$$

where, $\beta$ is the nonlinear absorption coefficient, $I_{0}$ is the light intensity at the focus, $L_{\mathrm{eff}}=\left(1-\mathrm{e}^{\alpha_{0} L}\right) / \alpha_{0}$ is the effective length of the sample, $\alpha_{0}$ is the linear absorption coefficient, $L$ is the thickness of the sample, $z_{0}=\pi \omega_{0}^{2} / \lambda$ is the Rayleigh length of the beam, $\omega_{0}$ is the beam waist, $\lambda$ is the wavelength, and $z$ is the Z-scan displacement. The value of $\beta$ was fitted to be $-1.09 \times 10^{-13} \mathrm{~m} \mathrm{~W}^{-1}$. Furthermore, the imaginary part of the third-order nonlinear optical susceptibility $\left(\operatorname{Im} \chi^{(3)}\right)$ can be calculated using the following formula: ${ }^{29}$

$$
\operatorname{Im} \chi^{(3)}=\frac{2 \varepsilon_{0} c^{2} n_{0}^{2}}{3 \omega} \beta
$$

where $\varepsilon_{0}$ is the vacuum permittivity, $c$ is the speed of light in vacuum, $n_{0}$ is the linear index and $\omega$ is the angular frequence. The calculated $\operatorname{Im} \chi^{(3)}$ is $-1.49 \times 10^{-22} \mathrm{~m}^{2} \mathrm{~V}^{-2}$. The closeaperture Z-scan results divided by the corresponding openaperture Z-scan results are shown in Fig. 2(d), and the normalized transmittance was fitted using the following formula:

$$
T_{\text {norm }}(z)=1+\frac{4 \Delta \Phi x^{2}}{\left(x^{2}+9\right)\left(x^{2}+1\right)}
$$

where, $\Delta \Phi=k n_{2} I_{0} L_{\text {eff }}, k$ is the wave vector, $n_{2}$ is the nonlinear refractive index and $x=z / z_{0}$. The value of $n_{2}$ was calculated to be $3.1 \times 10^{-20} \mathrm{~m}^{2} \mathrm{~W}^{-1}$. Furthermore, the real part of the thirdorder nonlinear optical susceptibility $\left(\operatorname{Re} \chi^{(3)}\right)$ was determined to be $1.01 \times 10^{-22} \mathrm{~m}^{2} \mathrm{~V}^{-2}$ using the formula: ${ }^{29}$

$$
\operatorname{Re} \chi^{(3)}=\frac{4 \varepsilon_{0} c n_{0}^{2}}{3} n_{2}
$$

\subsection{Laser performance of BP and stability test}

The experimental setup for the Yb-doped all-fiber ring laser based on BP-SA is depicted in Fig. 3(a). The fiber laser was pumped by a single-mode laser diode centered at $976 \mathrm{~nm}$ with a maximum pump output of $780 \mathrm{~mW}$. The pump light was coupled in the ring cavity via a broadband 976/1064 nm wavelength division multiplexing. The gain medium was a piece of 2 m-long Yb-doped fiber, with a core diameter of $6 \mu \mathrm{m}$ and a cladding diameter of $125 \mu \mathrm{m}$, and its absorption coefficient was about $12 \mathrm{~dB} \mathrm{~m}^{-1}$ and NA 0.12 . A polarization insensitive optical isolator centering at $1064 \mathrm{~nm}$ was inserted into the ring cavity to ensure unidirectional light propagation. A band-pass optical filter was adopted after the PI-ISO. To optimize the polarization state inside the cavity, a polarization controller was used. The SA was adsorbed on a fiber ferrule adapter. The BP-SA was adopted in the ring cavity to induce Q-switched operation. A coupler with $20 \%$ output was employed to output the intracavity energy. The output power was further divided into three parts to enable the simultaneous measurement of output power (15\%), optical spectrum (15\%) and pulse characteristic (70\%).

Based on the above experimental setup, the stable Qswitched laser pulse could be achieved when the pump power increased to $96 \mathrm{~mW}$. As is shown in Fig. 3(b), the output power increased linearly with an increase in pump power. The maximum output power was $12.6 \mathrm{~mW}$ at the pump power of 210 $\mathrm{mW}$. When the pump power increased above $210 \mathrm{~mW}$, the pulse operation could not be achieved, corresponding to the
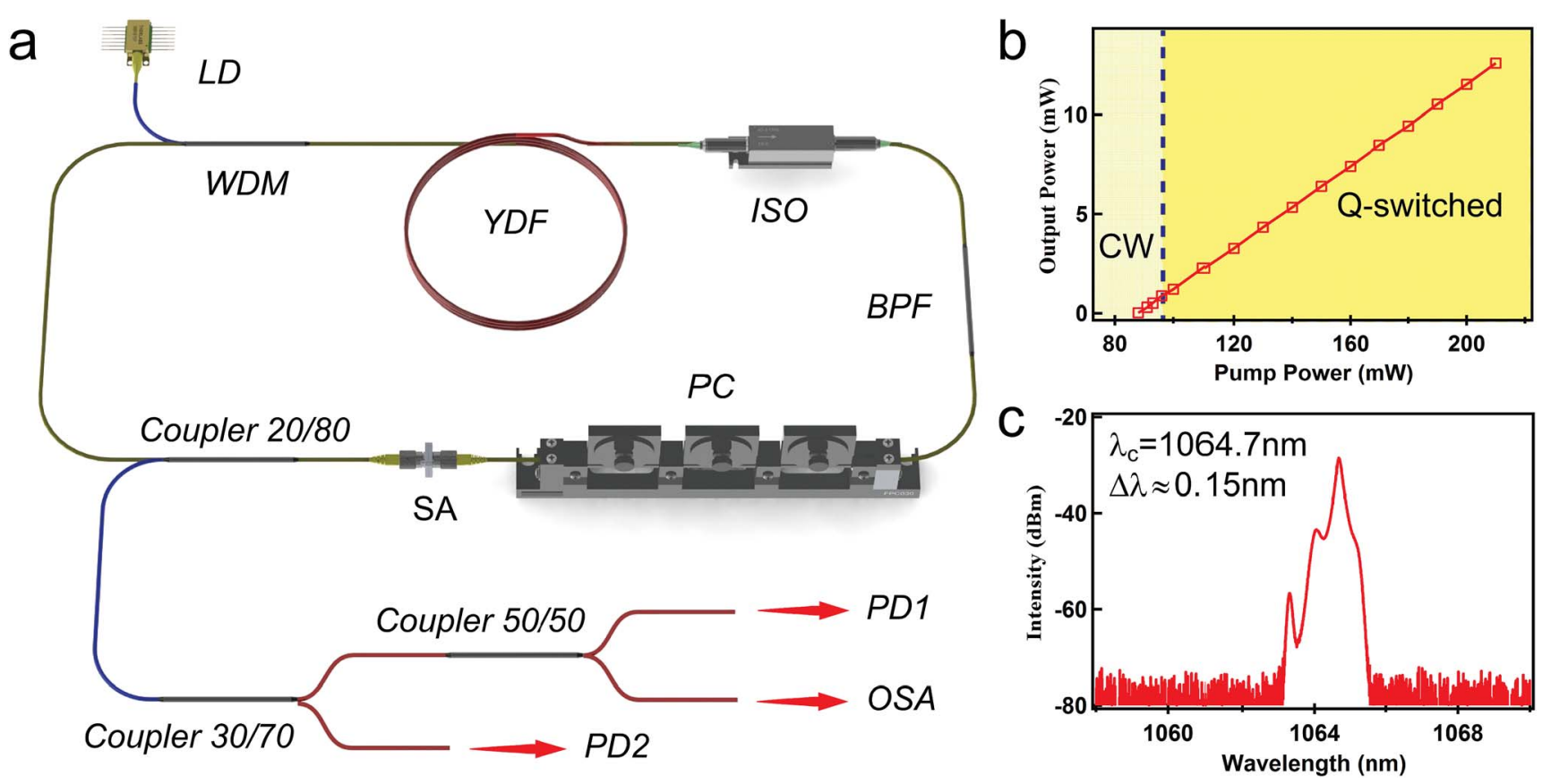

Fig. 3 (a) Experimental setup for the Yb-doped all-fiber ring laser based on BP-SA. LD: laser diode; WDM: wavelength division multiplexing; YDF: $\mathrm{Yb}$-doped fiber; PI-ISO: polarization-insensitive isolator; BPF: band-pass filter; PC: polarization controller; OSA: optical spectrum analyser; and PD: photodetector. (b) Relation between output power and input power and (c) spectrum of the laser. 
continuous wave output. However, when the pump power decreased to below $210 \mathrm{~mW}$, the Q-switched pulse could be observed again, which indicated that the BP-SA was not destroyed at a high pump power. The slope efficiency and optical-to-optical conversion efficiency of the laser cavity were about $11.1 \%$ and $6 \%$, respectively. As mentioned above, the SA was adsorbed on a fiber ferrule adapter to insert the cavity, which may bring additional optical loss into the cavity. Also, at the output, two additional couplers were connected, which can also bring additional insertion loss. The conversion efficiency can be enhanced if tapered fiber coated with BP-SA is employed or the cavity elements are optimized further.

The pump power was set to $150 \mathrm{~mW}$ to observe the pulse characteristics. Fig. 3(c) shows the spectrum of the output Qswitched pulse laser, which centers at $1064.7 \mathrm{~nm}$ with a $3 \mathrm{~dB}$ bandwidth of $0.15 \mathrm{~nm}$. Fig. 4(a) shows the pulse train with a pulse interval of $58.3 \mu \mathrm{s}$, corresponding to a repetition rate of 17.2 kHz. The pulse duration is shown in Fig. 4(b). The pulse envelope was fitted with a Gaussian function. The radio frequency spectrum is illustrated in Fig. 4(c). The fundamental frequency is $17.2 \mathrm{kHz}$ with a signal to noise ratio of $35 \mathrm{~dB}$, which indicates that the Q-switched pulse operation is quite stable.

The oscilloscopic traces at different pump power are shown in Fig. 3(a). With an increase in the pump power, the pulse interval decreased, and the pulse repetition rate increased. Fig. 4(b) depicts the repetition rate and pulse duration of the output laser as a function of pump power. As the pump power increased from $100 \mathrm{~mW}$ to $210 \mathrm{~mW}$, the repetition rate increased from $8.9 \mathrm{kHz}$ to $32.3 \mathrm{kHz}$. Although the pulse duration decreased from $14.18 \mu \mathrm{s}$ to $4.93 \mu \mathrm{s}$, the pulse duration showed a slight increase above the pump power of $180 \mathrm{~mW}$. This may be due to the over-saturation of BP-SA at the high pump power. In this case, the BP-SA could not respond instantaneously, thus resulting in the broadening of the pulse duration. This further led to a decrease in peak power, possibly when the pump power approached the maximum value. The pulse energy versus the pump power is also shown in Fig. 5(c). The maximum pulse energy of 450.14 nJ was achieved, corresponding to a maximum peak power of $90.03 \mathrm{~mW}$.

We also tested the stability of the Q-switched pulse. The pulse laser could operate stably for at least 69 days. Fig. 6 shows the typical stability test results under the pump power of 150 mW. From Fig. 6(a)-(c), we can see that the spectrum was quite stable. The central wavelength of $1064.7 \mathrm{~nm}$ hardly changed. The fluctuation of output power, repetition rate, and pulse duration from the average values were less than $4.19 \%, 1.23 \%$, and $3.98 \%$, respectively (Fig. 6(d) and (e)). In the early and late stages of 69 days, we tested the stability of the pulse laser for 4
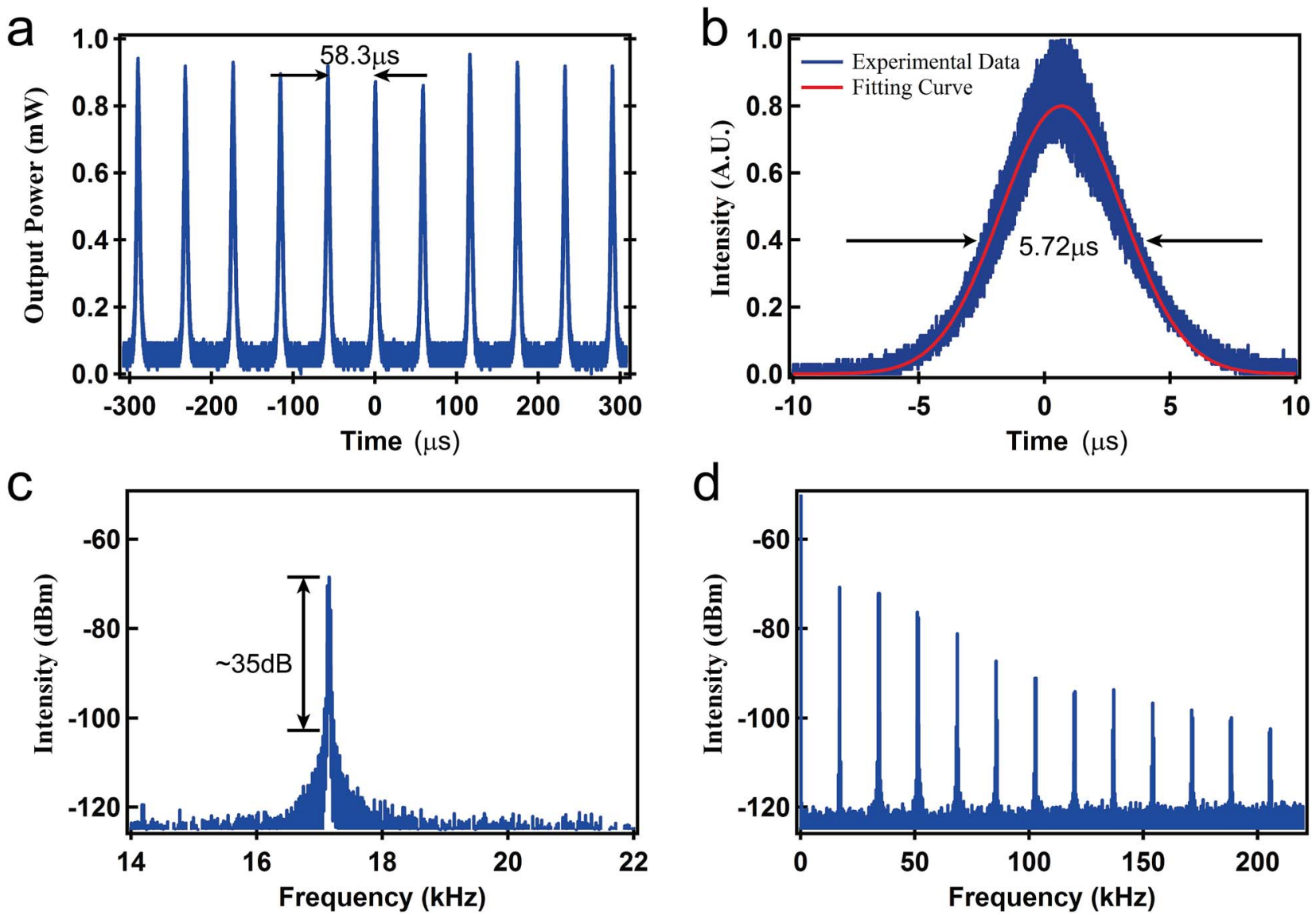

Fig. 4 Output laser properties under pump power of $150 \mathrm{~mW}$ : (a) pulse train, (b) pulse duration, (c) radio frequency spectrum, and (d) radio frequency spectrum in the frequency range of $200 \mathrm{kHz}$. 

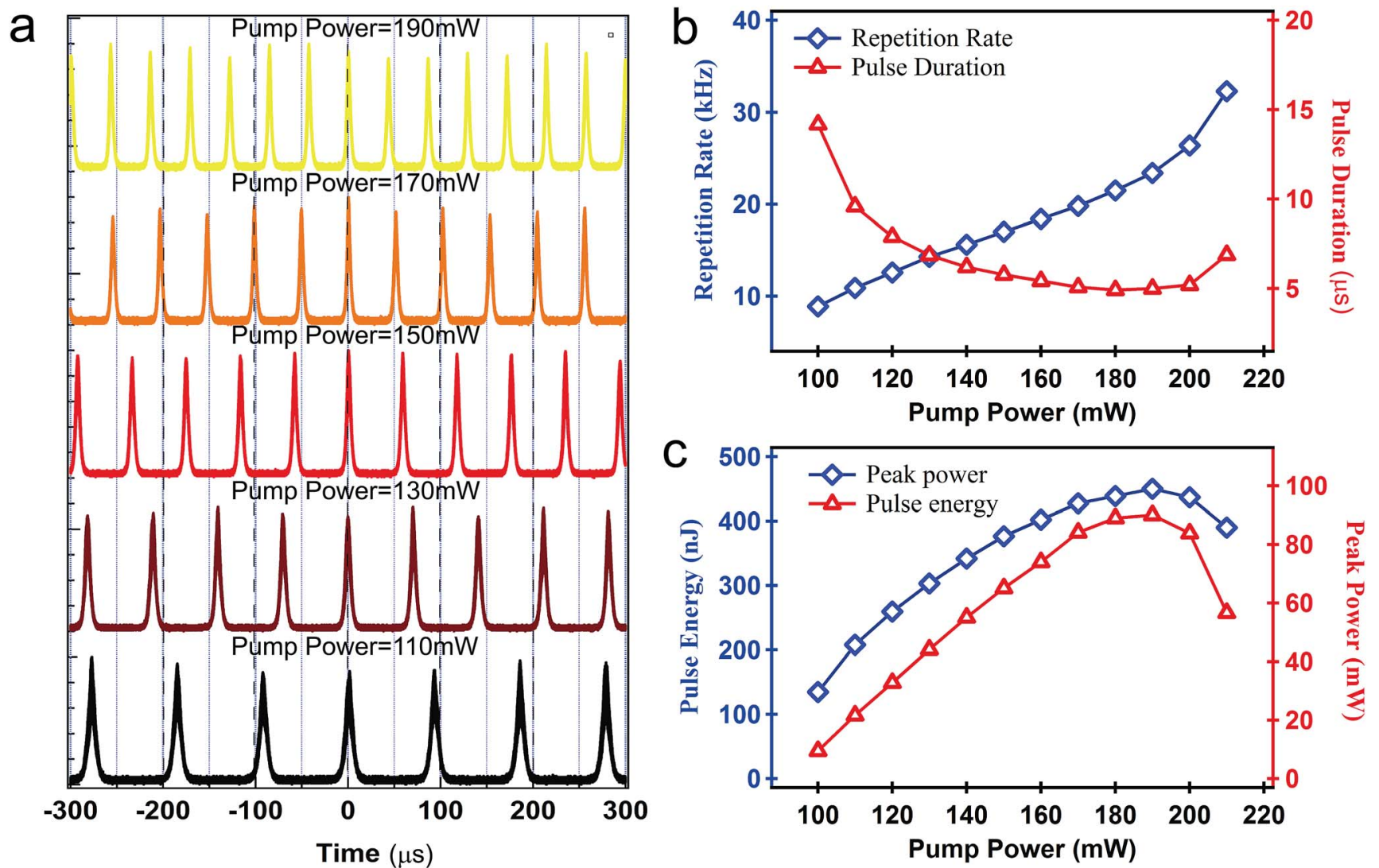

Fig. 5 (a) Oscilloscopic traces at different pump powers, (b) repetition rate and pulse duration versus pump power and (c) pulse energy and peak power versus pump power.

hours each day. The experimental results indicate that the pulse was also quite stable in single operation (see details in ESI†). According to the structure of $\mathrm{BP}$, the outermost layer of $\mathrm{BP}$ has five electrons, so the surface of $\mathrm{BP}$ has free charges, making it easy to be oxidized and then degrade in the environment, where water and oxygen coexist. In our work, BP nanoflakes were directly filtered into a thin film after electrochemical exfoliation in a $\mathrm{TBA}^{+}$solution, exhibiting excellent stability in air. On the
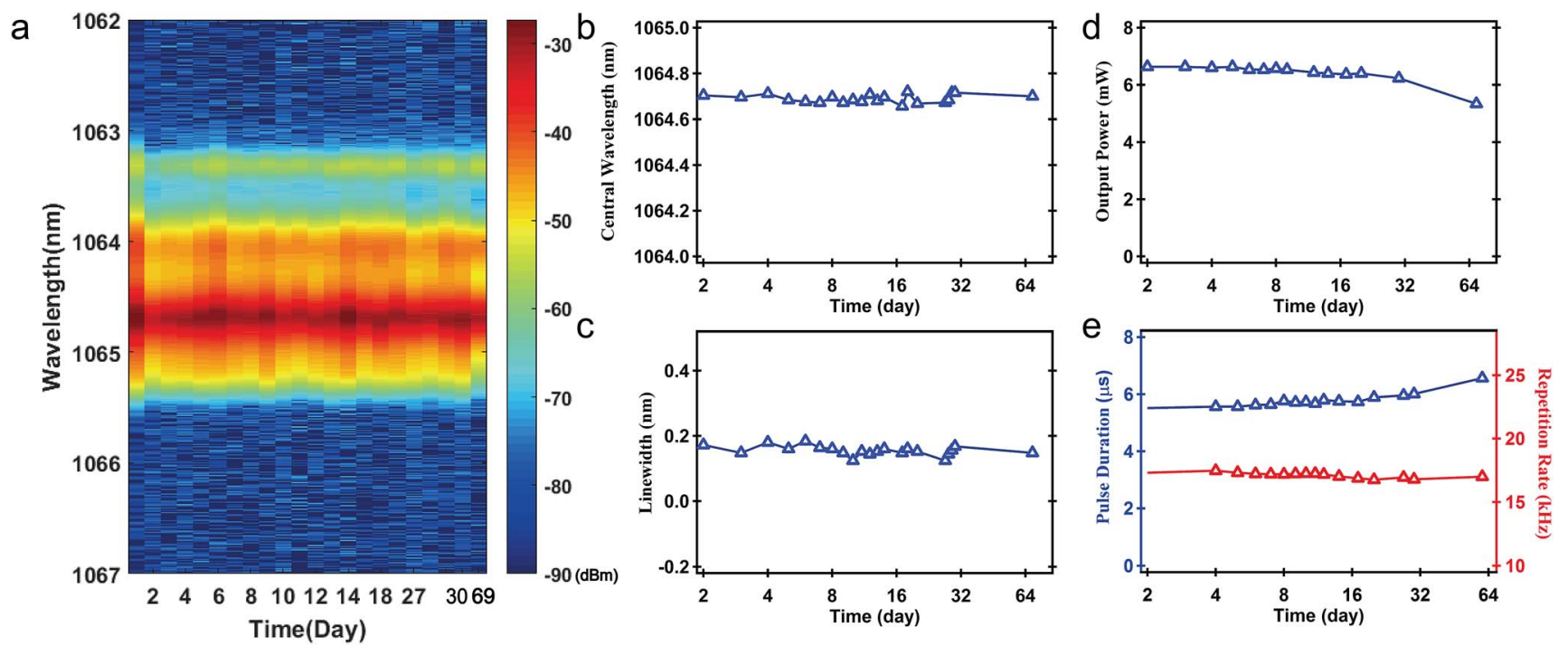

Fig. 6 Stability results of the pulse laser: (a) emission spectrum, (b) central wavelength, (c) full width at half maximum of the central wavelength, (d) output power, and (e) repetition rate and pulse duration at different time intervals. 
one hand, the positive charges of $\mathrm{TBA}^{+}$can neutralize the negative charges of BP itself, which can improve the stability of the BP nanoflakes. On the other hand, the electrolyte is not volatile and has a relatively high boiling point $\left(242^{\circ} \mathrm{C}\right)$. During the filtration process, a thin layer of residual solvent adhered to the BP surface, behaving as an isolating barrier, which could protect the BP flakes from surface oxidation. Additionally, encapsulating BP-SA in two fiber ferrules weakened the interactions between SA and air or water vapor, which further enhanced its long-term stability. To the best of our knowledge, our pulse laser displays the best stability performance compared with that reported previously.

In this work, the mode-locked operation was not achieved, which was due to several aspects. First, the cavity loss was too high to achieve the power level for mode-locked operation. The cavity loss was possibly from the cavity elements, fiber ferrule adapter and SA. Besides, the mode-locked operation needs to achieve phase locking among a number of longitudinal modes. However, the $3 \mathrm{~dB}$ bandwidth of BPF was less than $0.2 \mathrm{~nm}$ in this work, which is too small to achieve mode-locked operation. Furthermore, the thickness of the BP-SA sample could affect the modulation depth, which is a pivotal factor that determines the mode-locked operation. Thus, the mode-locked pulse can be expected by reducing the cavity loss, employing a broadband BPF and improving the BP-SA thickness.

\section{Conclusions}

In conclusion, a passively Q-switched pulse laser was demonstrated with a BP-SA. Compared to liquid phase exfoliation technology or the mechanical exfoliation method, larger-size and more excellent time stability were achieved in the BP fabricated by a modified electrochemical delamination exfoliation. Based on this BP-SA, the pulse laser can operate stably for at least 69 days. The maximum average output power was 12.6 $\mathrm{mW}$ with an $11.1 \%$ slope efficiency and $6 \%$ optical-to-optical conversion efficiency. The pulse repetition rate could be tuned from $8.9 \mathrm{kHz}$ to $32.3 \mathrm{kHz}$, while the maximum pulse energy of $450.14 \mathrm{~nJ}$ could be achieved, which corresponds to the maximum peak power of $90.03 \mathrm{~mW}$. The experimental results reveal that our BP can be adopted as an ultra-stable SA for application in pulse lasers.

\section{Conflicts of interest}

There are no conflicts to declare.

\section{Acknowledgements}

This project was supported by the National Nature Science Foundation of China (Grant No. 61705265), Shenzhen Basic Research Project of Science and Technology (No. JCYJ20170302142433007), the CAS Key Laboratory of Nano-Bio Interface (17GJ02), the Natural Science Foundation of Hunan province (2018JJ3610), and the Open Research Fund of State Key Laboratory of Pulsed Power Laser Technology, the Electronic Engineering Institute (SKL2016KF04).

\section{References}

1 Q. L. Bao, H. Zhang, Y. Wang, Z. H. Ni, Y. L. Yan, Z. X. Shen, K. P. Loh and D. Y. Tang, Adv. Funct. Mater., 2009, 19, 30773083.

2 T. Hasan, Z. P. Sun, F. Q. Wang, F. Bonaccorso, P. H. Tan, A. G. Rozhin and A. C. Ferrari, Adv. Mater., 2009, 21, 38743899.

3 W. Liu, L. Pang, H. Han, K. Bi, M. Lei and Z. Wei, Nanoscale, 2017, 9, 5806-5811.

4 S. Zhang, N. Dong, N. McEvoy, M. O'Brien, S. Winters, N. C. Berner, C. Yim, Y. Li, X. Zhang, Z. Chen, L. Zhang, G. S. Duesberg and J. Wang, ACS Nano, 2015, 9, 7142-7150.

5 Z. Luo, Y. Huang, J. Weng, H. Cheng, Z. Lin, B. Xu, Z. Cai and H. Xu, Opt. Express, 2013, 21, 29516-29522.

6 Y. Meng, G. Semaan, M. Salhi, A. Niang, K. Guesmi, Z. Luo and F. Sanchez, Opt. Express, 2015, 23, 23053-23058.

7 Z. Luo, M. Liu, H. Liu, X. Zheng, A. Luo, C. Zhao, H. Zhang, S. Wen and W. Xu, Opt. Lett., 2013, 38, 5212-5215.

8 Y. Chen, G. Jiang, S. Chen, Z. Guo, X. Yu, C. Zhao, H. Zhang, Q. Bao, S. Wen, D. Tang and D. Fan, Opt. Express, 2015, 23, 12823-12833.

9 Y. Xu, X. Jiang, Y. Ge, Z. Guo, Z. Zeng, Q. Xu, H. Zhang, X. Yu and D. Fan, J. Mater. Chem. C, 2017, 5, 3007-3013.

10 H. O. Churchill and P. Jarillo-Herrero, Nat. Nanotechnol., 2014, 9, 330-331.

11 A. N. Rudenko and M. I. Katsnelson, Phys. Rev. B, 2014, 89, 201408.

12 V. Tran, R. Soklaski, Y. Liang and L. Yang, Phys. Rev. B, 2014, 89, 235319.

13 K. Wu, B. Chen, X. Zhang, S. Zhang, C. Guo, C. Li, P. Xiao, J. Wang, L. Zhou, W. Zou and J. Chen, Opt. Commun., 2018, 406, 214-229.

14 F. Zhang, Z. Wu, Z. Wang, D. Wang, S. Wang and X. Xu, RSC Adv., 2016, 6, 20027-20033.

15 Z. Luo, M. Liu, Z. Guo, X. Jiang, A. Luo, C. Zhao, X. Yu, W. Xu and H. Zhang, Opt. Express, 2015, 23, 20030-20039.

16 M. B. Hisyam, M. F. M. Rusdi, A. A. Latiff and S. W. Harun, IEEE J. Sel. Top. Quantum Electron., 2017, 23, 39-43.

17 H. Ahmad, M. A. M. Salim, K. Thambiratnam, S. F. Norizan and S. W. Harun, Laser Phys. Lett., 2016, 13, 095103.

18 F. A. A. Rashid, S. R. Azzuhri, M. A. M. Salim, R. A. Shaharuddin, M. A. Ismail, M. F. Ismail, M. Z. A. Razak and H. Ahmad, Laser Phys. Lett., 2016, 13, 085102.

19 V. Eswaraiah, Q. Zeng, Y. Long and Z. Liu, Small, 2016, 12, 3480-3502.

20 J. Sotor, G. Sobon, W. Macherzynski, P. Paletko and K. M. Abramski, Appl. Phys. Lett., 2015, 107, 051108.

21 K. X. Huang, B. L. Lu, D. Li, X. Y. Qi, H. W. Chen, N. Wang, Z. R. Wen and J. T. Bai, Appl. Opt., 2017, 56, 6427-6431.

22 E. I. Ismail, N. A. Kadir, A. A. Latiff, H. Ahmad and S. W. Harun, RSC Adv., 2016, 6, 72692-72697.

23 Y. Ge, S. Chen, Y. Xu, Z. He, Z. Liang, Y. Chen, Y. Song, D. Fan, K. Zhang and H. Zhang, J. Mater. Chem. C, 2017, 5, 6129-6135. 
24 J. O. Island, G. A. Steele, H. S. J. v. d. Zant and A. CastellanosGomez, 2D Materials, 2015, 2, 011002.

25 S. Yang, K. Zhang, A. G. Ricciardulli, P. Zhang, Z. Liao, M. R. Lohe, E. Zschech, P. W. M. Blom, W. Pisula and K. Müllen, Angew. Chem., 2018, 130, 4767-4771.

26 X. Yijun, Y. Jian, F. Linfeng, W. Xinliang, B. Qiaoliang, W. Yu, Z. Kai and Z. Yuegang, Small, 2016, 12, 5000-5007.
27 Z. Guo, H. Zhang, S. Lu, Z. Wang, S. Tang, J. Shao, Z. Sun, H. Xie, H. Wang and X. Yu, Adv. Funct. Mater., 2016, 25, 6996-7002.

28 M. Sheik-Bahae, A. A. Said, T.-H. Wei, D. J. Hagan and E. W. Van Stryland, IEEE J. Quantum Electron., 1990, 26, 760-769.

29 J. Cheng, J. Hao, H. Liu, J. Li, J. Li, X. Zhu, X. Lin, K. Wang and T. He, ACS Nano, 2018, 12, 5341-5350. 